\title{
Basis Expansion Leaping: A New Method to Solve the Time-Dependent Schrödinger Equation for Molecular Quantum Dynamics
}

\author{
Werner Koch* and Terry J. Frankcombe ${ }^{\dagger}$ \\ Research School of Chemistry, Australian National University, Australian Capital Territory 0200, Australia
} (Received 29 August 2012; revised manuscript received 24 February 2013; published 26 June 2013)

\begin{abstract}
A wide variety of molecular systems that have recently come into the reach of experimental and theoretical investigation is dominated by quantum phenomena. However, even state of the art quantum propagation techniques are either unsuitable for general application to molecular systems with strong interference and tunneling characteristics or are computationally prohibitive for systems with more than a few degrees of freedom. In this Letter, we introduce a novel quantum propagation technique with wide applicability, controllable accuracy, and efficient utilization of computational resources. Its performance is validated for tunneling and dissociating systems with 1,2, and 3 degrees of freedom, and the scaling behavior with respect to system dimensionality and requested accuracy is discussed.
\end{abstract}

DOI: 10.1103/PhysRevLett.110.263202

PACS numbers: $34.10 .+\mathrm{x}, 03.65 .-\mathrm{w}, 82.20 . \mathrm{Wt}$

In recent years, the frontiers of physics and chemistry have advanced to the point where the understanding of quantum molecular processes has become critical and classical or semiclassical techniques are no longer adequate for reproducing observed effects. Consequently, ever more refined numerical methods are being developed to compute the quantum dynamics of molecular systems [1-5]. One exceptionally successful method in terms of balancing computational requirements and accuracy is the multiconfiguration time-dependent Hartree (MCTDH) method [6,7]. The variational multiconfiguration Gaussian (vMCG) approach is a descendant of MCTDH that uses localized Gaussian functions as a basis set $[8,9]$. This is particularly suited to modeling the nuclear wave functions of molecular systems as they typically contain strongly localized components. The localized nature of the Gaussian basis functions tremendously reduces the cost of the potential energy surface (PES) computation in direct dynamics [10] or interpolating approaches [11] when combined with the local harmonic approximation of the potential, where the Taylor expansion of the potential is truncated at second order. vMCG formally scales much better with dimensionality than grid based methods. It turns out, however, that processes that involve strongly interfering components of the wave packet, such as tunneling scenarios, pose special numerical problems that are difficult if not impossible to circumvent with vMCG.

In this Letter, we present a novel approach to modeling such challenging nuclear molecular dynamics processes with controllable accuracy. It inherits the advantages of localized basis functions and variational propagation from vMCG but has lower computational cost and can therefore handle the large basis sets that are necessary to accurately model tunneling dynamics.

In vMCG, the wave packet is expanded in a set of timedependent Gaussian wave packets (GWPs) whose parameters evolve according to variational equations derived from the Schrödinger equation. Most applications of vMCG employ fixed width Gaussian functions since the use of variable width Gaussians increases classicality of the GWP trajectories and incurs numerical problems with the propagation [12]. In principle, this approach results in a variationally optimal representation of the wave packet that would allow modeling of quantum phenomena with a minimal number of GWPs. However, the Gaussian basis is overcomplete and nonorthogonal, which can lead to severe numerical instabilities. Unfortunately, this is especially prevalent in tunneling scenarios as individual Gaussians have to approach each other to accurately represent interference patterns. Furthermore, a very large number of narrow, fixed width Gaussians is needed to accommodate a wave packet with many fine details when interacting with a barrier as well as where it has a wide extent before and after the interaction. The propagation of the Gaussian parameters as given in Ref. [9] also requires the simultaneous propagation of a matrix that transforms the GWPs into "single particle functions." Thus, the number of differential equations that need to be integrated scales quadratically with the number of GWPs. With the large number of GWPs needed for tunneling scenarios, this computational cost becomes prohibitive.

One method that has been used in an attempt to avoid problems in integrating the evolution of the GWP basis is ab initio multiple spawning [13,14], in which the centers of the Gaussians are taken to follow classical paths. However, in this approach, the basis set evolves completely independently of adiabatic quantum effects such as quantum tunneling and interference, reducing the effectiveness of the method in resolving such effects.

The approach described in this Letter eliminates these issues by treating the overcompleteness of the Gaussian basis functions as a resource rather than a numerical difficulty. The basis being overcomplete has two key consequences: there are many ways to express the same wave 
function, and wave functions can be accurately propagated for short times without changing the basis at all. We use both of these properties in our new method, which comes down to performing propagations for short times without changing the basis of Gaussian functions, interspersed with reexpressing the propagated wave function with a new set of Gaussians adapted to the evolved wave function. Similar reexpression of Gaussian wave packets has been used in "matching pursuit" schemes for split operator propagators $[15,16]$. However, we demonstrate that a (potentially costly) reexpansion into a new basis is not necessary at every time step. We shall henceforth call this new method the basis expansion leaping multiconfiguration Gaussian (BEL MCG) propagation. A significant difference between BEL MCG and both ab initio multiple spawning and vMCG is that BEL MCG does not attempt to continuously update the existing basis set for propagation at later times, and so contains no equations of motion for basis functions.

In BEL MCG, the nuclear wave function is expanded in a set of Gaussians that remain stationary between reexpressions (all quantities given are in atomic units and $\hbar=1)$ :

$$
\Psi(\mathbf{x}, t)=\sum_{i=1}^{N} A_{i}(t) g_{\mathbf{Q}_{i}, \boldsymbol{\sigma}_{i}, \mathbf{P}_{i}}(\mathbf{x}),
$$

with $\mathbf{x}$ the vector of nuclear coordinates and

$g_{\mathbf{Q}_{i}, \boldsymbol{\sigma}_{i}, \mathbf{P}_{i}}(\mathbf{x})=\exp \left\{\left(\mathbf{x}-\mathbf{Q}_{i}\right)^{T}\left[a_{i}\left(\mathbf{x}-\mathbf{Q}_{i}\right)+\mathrm{i} \mathbf{P}_{i}\right]+\phi_{i}\right\}$,

where $\mathbf{Q}_{i}$ and $\mathbf{P}_{i}$ are vectors of position and momentum parameters, $a_{i}$ is a diagonal matrix whose diagonal elements $a_{i, j j}=-\left(2 \sigma_{i, j}\right)^{-2}$ are defined by the elements of the vector of widths $\boldsymbol{\sigma}_{i}$, and $\phi_{i}$ is a real normalization parameter.

In the subspace spanned by these Gaussians, the time evolution of the coefficients according to the Schrödinger equation is provided by the Dirac-Frenkel variational principle $\left\langle\delta \Psi\left|\hat{H}-\mathrm{i} \partial_{t}\right| \Psi\right\rangle=0[17,18]$, with the Hamiltonian operator $\hat{H}$, yielding

$$
\text { i } \dot{\mathbf{A}}(t)=S^{-1} H \mathbf{A}(t),
$$

where $S_{i j}=\left\langle g_{i} \mid g_{j}\right\rangle$ is the overlap matrix of the Gaussians, and $H_{i j}=\left\langle g_{i}|\hat{H}| g_{j}\right\rangle$ is the Hamiltonian matrix. The potential part of the Hamiltonian will be evaluated in the local harmonic approximation. For time-independent Hamiltonians, Eq. (3) is a set of coupled differential equations with constant coefficients that may be solved with a wide variety of methods. For the example systems discussed in this Letter, numerical adaptive step size integration has proven efficient and robust (cf. [19]).

Before the wave packet evolves beyond the space spanned by the current set of Gaussians, a reexpansion in a new set needs to be performed to accommodate the changed state. The reexpansion produces a completely new set of Gaussians, adapted to the current wave function. The BEL MCG's performance does not critically depend on the specific way of choosing the new expansion, beyond requiring that the wave function can be faithfully reproduced in the new basis set (that is, that one can achieve a negligible deviation from the wave packet expressed in the previous set of Gaussians). The procedure described in Ref. [16] scans the entire Gaussian parameter space with a Monte Carlo sampling. While this allows for reexpansions with a very low deviation, it is computationally expensive and constructs unnecessarily complex expressions of the wave packet, again leading to numerical problems. Fortunately, the redundancy of the GWP set is so large that there are many configurations of Gaussians that are equally well suited to expressing the wave function. The position, momentum, and width parameters can be selected separately and chosen such that auxiliary optimization criteria are satisfied.

The following iterative procedure for assigning the Gaussian function parameters has proven flexible and robust. It is controlled by a single accuracy parameter $\varepsilon$. In the limit of $\varepsilon \rightarrow 0$, BEL MCG converges toward a full expansion of the wave packet and thus reproduces the quantum dynamics of the system exactly.

When performing a reexpansion, the new set of Gaussian functions depends on the previous set of Gaussians only indirectly, through the form of the evolved $\Psi_{\text {old }}$ expressed in the previous set of basis functions. Basis functions are added sequentially to the new basis set, starting from an empty set. For the $i$ th new Gaussian, (1) Determine the position $\mathbf{Q}_{i}=\max _{\mathbf{Q}} f_{i}(\mathbf{Q})$ with

$f_{i}(\mathbf{Q})=\Theta\left[\left|\Psi_{\text {old }}(\mathbf{Q})\right|^{2}-\varepsilon\right] \sum_{k=1}^{i-1}\left\|\mathbf{Q}-\mathbf{Q}_{k}\right\|+\omega\left|\delta \Psi_{i-1}(\mathbf{Q})\right|^{2}$,

where $\Theta[\cdot]$ is the Heaviside step function and $\delta \Psi_{i-1}(\mathbf{Q})=$ $\Psi_{\text {old }}(\mathbf{Q})-\Psi_{i-1}(\mathbf{Q})$ is the remaining deviation between the wave function before the reassignment $\Psi_{\text {old }}$ and the wave function composed of the first $i-1$ new Gaussians $\Psi_{i-1} . f_{i}(\mathbf{Q})$ is sufficiently smooth and simple for efficient simplex maximization with the Nelder-Mead method [20]. It ensures that Gaussians are placed in regions that contribute significantly to the probability distribution $(\Theta[\cdot])$ while spacing them as uniformly as possible $\left(\sum\|\cdot\|\right)$ and targeting remaining deviations (last term). A uniform spacing results in lower basis set overlaps and higher numerical stability of the subsequent propagation step. The factor $\omega$, whose units depend on the dimensionality of the problem, adjusts the balance between these considerations. (2) Adjust the widths $\boldsymbol{\sigma}_{i}$ such that

$$
\left|\tilde{V}\left(\mathbf{Q}_{i}+\mathbf{e}_{j} \sigma_{i, j}\right)-V\left(\mathbf{Q}_{i}+\mathbf{e}_{j} \sigma_{i, j}\right)\right| \leq \varepsilon
$$


for all degrees of freedom $j$, with

$$
\tilde{V}\left(\mathbf{Q}_{i}+\mathbf{e}_{j} \sigma_{i, j}\right)=V\left(\mathbf{Q}_{i}\right)+\left.\sigma_{i, j} \frac{\partial V}{\partial x_{j}}\right|_{\mathbf{Q}_{i}}+\left.\frac{\sigma_{i, j}^{2}}{2} \frac{\partial^{2} V}{\partial x_{j}^{2}}\right|_{\mathbf{Q}_{i}}
$$

the second order Taylor expansion of the potential at $\mathbf{Q}_{i}$ along the cardinal direction $\mathbf{e}_{j}$. This condition ensures an acceptably low error is incurred by the local Harmonic approximation. In practice, this is achieved by sequential halving of $\sigma_{i, j}$ from a default value until Eq. (5) is obeyed, for each $j$. (3) Determine the $\operatorname{momentum} \mathbf{P}_{i}=\max _{\mathbf{P}} f_{i}^{\prime}(\mathbf{P})$ with

$$
f_{i}^{\prime}(\mathbf{P})=\left\langle g_{\mathbf{Q}_{i}, \boldsymbol{\sigma}_{i}, \mathbf{P}} \mid \delta \Psi_{i-1}\right\rangle .
$$

The Nelder-Mead method is used again here. This step is analogous to the procedure described in Ref. [16]. After this maximization step, the initial expansion coefficient in Eq. (1) is determined by $A_{i}=f_{i}^{\prime}\left(\mathbf{P}_{i}\right)$.

Note that the optimizations in steps 1 and 3 need not be to extremely high tolerance. This is another manifestation of the overcompleteness of the Gaussian function basis.

While it remains numerically significant, the magnitude of $\left\langle\delta \Psi_{i} \mid \delta \Psi_{i}\right\rangle$ decreases as Gaussians are added with the procedure above. New Gaussians need to be added to the list until $\left\langle\delta \Psi_{i} \mid \delta \Psi_{i}\right\rangle<\varepsilon$. After that limit is reached, the procedure can optionally be continued to add a set of negligibly populated Gaussians. These additional functions can be used as sentinels to guard against propagating for too long before reexpression. A rising population of these Gaussians is one indication that the significantly populated states no longer span a suitable space for the wave packet and a reassignment is necessary.

With the new basis set assigned with negligible perturbation of the wave function, the matrices $S^{-1}$ and $H$ required for Eq. (3) are computed from the new parameter set $\left\{\mathbf{Q}_{i}, \boldsymbol{\sigma}_{i}, \mathbf{P}_{i}\right\}$ and propagation of the coefficients A continues.

Both the reassignment as well as the propagation scale as $\mathcal{O}\left(N^{2}\right)$ with the number of Gaussians $N$, whereas vMCG scales as $\mathcal{O}\left(N^{4}\right)$. Therefore, the much larger number of Gaussians required to correctly describe the wave packet while allowing for narrow widths in the vicinity of complex regions of the potential in tunneling scenarios can easily be accommodated. Adaptive assignment of Gaussian widths provides a further advantage over vMCG, in which the widths stay constant throughout the propagation irrespective of the nature of the PES in the vicinity of the basis function.

An important aspect of the basis set reexpression procedure is that the locations of the centers of the new Gaussians do not depend directly on the locations of the centers of the previous set of Gaussians. When the wave function is propagating into new regions of configuration space, the flexibility of the Gaussian basis allows probability density to migrate a short distance outside the domain of the Gaussian function centers. When reexpressed in an unbiased way, this density causes some of the new basis functions to be located in these regions that the wave function is propagating into. Similarly, regions of negligible probability density do not get populated with basis functions, allowing the domain of the basis functions to move through space with the wave function.

Clearly, accurate propagation requires a sufficiently high reexpression frequency, which depends on the details of the calculation. An adaptive algorithm is again used here. From an initial conservative (high) reexpression frequency, if the number of GWPs required to achieve the required reexpression accuracy $\varepsilon$ is below the user selected maximum, the reexpression frequency is decreased. Conversely, rewinding the propagation and increasing reexpression frequency is effective in maintaining reexpression accuracy. The reexpression frequency is thus automatically adjusted to suit the current complexity of the wave function.

To demonstrate the viability of the presented approach, one-, two-, and three-dimensional examples will be discussed that cover asymptotically free and bound barrier tunneling scenarios as well as realistic molecular evolution. The tunneling problems in particular present significant challenges that cannot be solved simply with vMCG. The initial wave packets for all systems are chosen to be of Gaussian form analogous to Eq. (2).

For transmission through a one-dimensional Eckart [21] barrier, with $\varepsilon=10^{-3}$, the BEL MCG method can readily calculate accurate transmission probabilities from 1 down to $10^{-3}$. A more stringent $1 \mathrm{D}$ test is transmission through a rounded rectangular barrier described by

$$
V_{1}(x)=\frac{D}{2}\{\tanh [\beta(x-\gamma)]-\tanh [\beta(x+\gamma)]\},
$$

with barrier height $D$, width $\gamma$, and steepness parameter $\beta$. It is more demanding than the Eckart barrier because its steepness requires much narrower Gaussian widths for accuracy in the local harmonic approximation, resulting in a higher number of basis functions for the same level of propagation accuracy.

Figure 1 shows a snapshot of the density of the propagated wave packet just after interacting with the barrier. Both the reflected part with the interference pattern as well as the rather smooth transmitted part are reproduced well with BEL MCG. The degree of convergence with respect to the deviation parameter $\varepsilon$ is shown. At most, 600 Gaussians were required. The quality of reproduction is similar for other times, through to the wave function no longer significantly interacting with the barrier.

As a 2D test problem, we use the potential

$$
V_{2}\left(x_{1}, x_{2}\right)=V_{1}\left(x_{1}\right)+k\left[\left(x_{1}^{2}+x_{2}^{2}\right)^{1 / 2}-\delta\right]^{2} \text {, }
$$

a harmonic ring with the rounded rectangular barrier superimposed on it. $\delta$ and $k$ denote the radius and the curvature of the ring, respectively. This potential is not separable and 


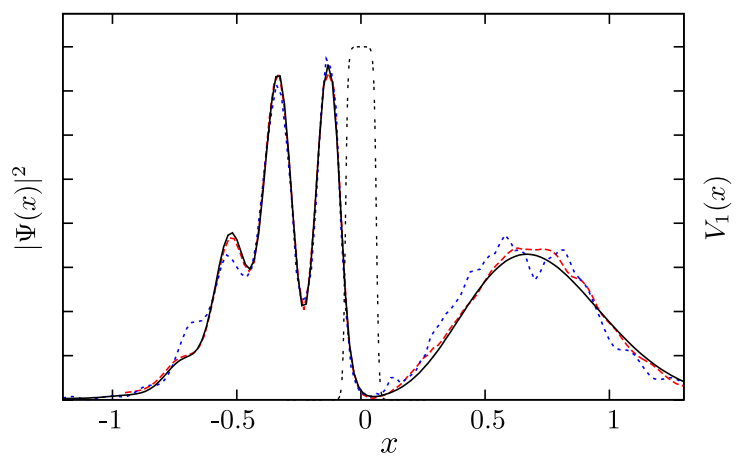

FIG. 1 (color online). Propagated density for rounded rectangular barrier (double-dashed line, right axis) with (all in atomic units) $D=30, \beta=100$, and $\gamma=0.0625$. The $\Psi(x, 0)$ parameters are $Q=-0.7, \sigma=0.0728$, and $P=25$. The mass is 10 . Density at $t=0.012$ is shown for exact calculation (solid black line) and BEL MCG with $\varepsilon=10^{-2}$ (dotted blue line) and $\varepsilon=10^{-3}$ (dashed red line).

anharmonic, presenting a challenging test case that incurs prohibitively large computational requirements when treated with vMCG.

Figure 2 shows the density of the wave packet just after interacting with the barrier. The distribution of the density along the potential ring with the transmitted part and the interference pattern of the reflected part are all excellently reproduced. As expected, more Gaussians are required for the same $\varepsilon$ than in the 1D case, with 800 Gaussians being required to maintain accuracy at the $\varepsilon=10^{-3}$ level. This example involved 276 reexpressions (cf. 12119 integration time steps). For comparison, a poorly converged, $100 \mathrm{GWP}$ vMCG calculation for the same wave packet requires twice the CPU time.

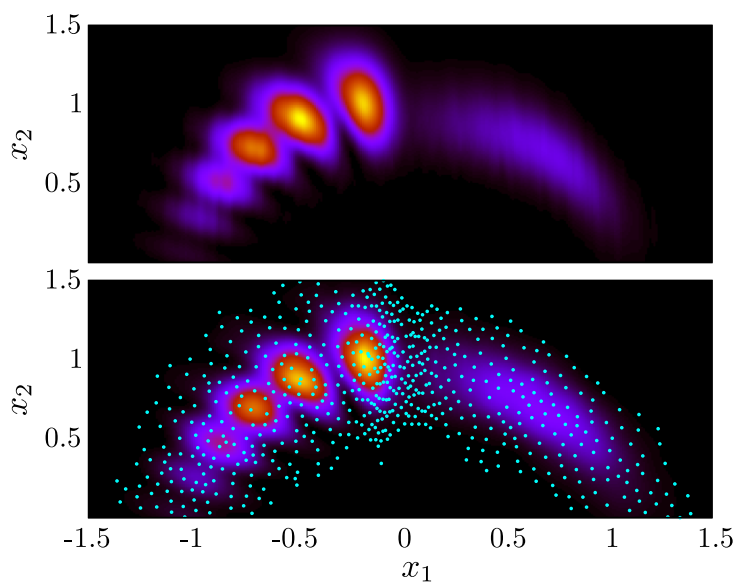

FIG. 2 (color online). Density propagated on $V_{2}$ with (all in atomic units) $D=15, \beta=30, \gamma=0.0625, k=20$, and $\delta=1$. The $\Psi(x, 0)$ parameters are $Q_{1}=-1, Q_{2}=0, \sigma_{1}=\sigma_{2}=0.1, P_{1}=0$, and $P_{2}=15$. The mass is 10 . Density at $t=0.055$ is shown for exact calculation (top panel) and BEL MCG with $\varepsilon=10^{-3}$ (bottom panel) with locations of Gaussian function centers (circles).
If a more integrated quantity such as barrier transmission probability is desired, a larger $\varepsilon$ and thus a much lower number of Gaussians are sufficient to obtain converged results with BEL MCG.

An application of BEL MCG to a more realistic problem, although not one involving tunneling, is the calculation of the photodissociation spectrum of $\mathrm{NOCl}$ on the $S_{1}$ surface. This problem has been studied in detail in the past and has been used for testing early in the development of new quantum dynamics methods $[11,22,23]$. We perform the 3D calculation in body-fixed Cartesian coordinates, using the same PES as in previous work. See Refs. [11,23] for details. A single populated Gaussian is used as the initial wave packet on the $S_{1}$ surface, and the photodissociation spectrum is calculated from the autocorrelation function of the wave function evolving in time.

Figure 3 shows the calculated photodissociation spectrum calculated with BEL MCG and with MCTDH using the same Hamiltonian. As $\varepsilon$ decreases, the calculated photodissociation spectrum converges toward the converged MCTDH result. Achieving the $\varepsilon=10^{-3}$ matching criterion required at most 1300 Gaussians. The shape and position of the adsorption feature is well reproduced, although at $\varepsilon=10^{-3}$ a small difference remains in the peak intensity.

These test systems demonstrate the BEL MCG's applicability to a variety of scenarios related to the nuclear motion of chemical systems on a single PES [24]. Convergence toward the correct result is shown for all of these systems. While the required number of Gaussians depends strongly on the type of system under investigation, the method is generally applicable to any time-independent potential surface. Generalization to fully coupled, multistate PESs is expected to be straightforward, but timevarying Hamiltonians lie outside the scope of BEL MCG. The reexpression procedure described in this Letter is

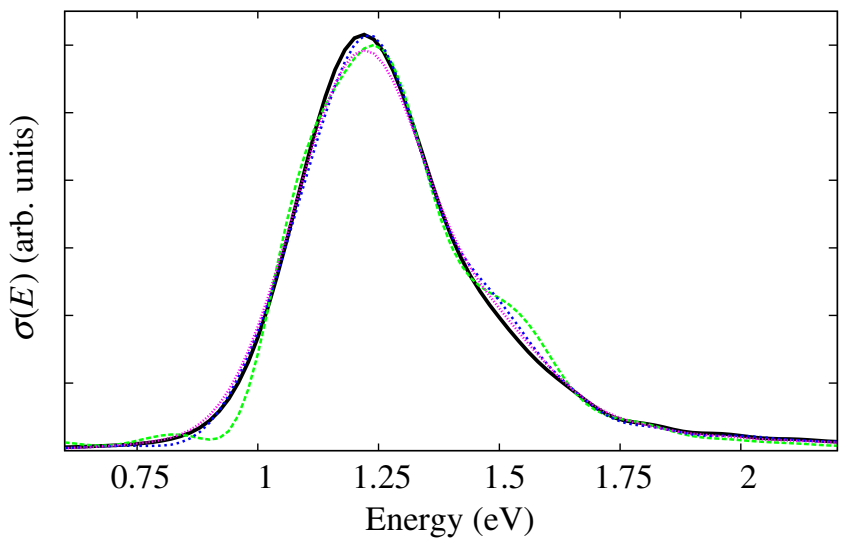

FIG. 3 (color online). Photodissociation spectrum of $\mathrm{NOCl}$ on $S_{1}$ calculated with MCTDH (solid black line) and BEL MCG with $\varepsilon=3 \times 10^{-2}$ (long-dashed green line), $\varepsilon=3 \times 10^{-3}$ (short-dashed blue line), and $\varepsilon=1 \times 10^{-3}$ (dotted magenta line). The wave function is propagated for $20 \mathrm{a} . \mathrm{u}$. 
efficient, robust, adapts automatically to the user selectable accuracy parameter, and only requires the PES evaluation at reassignment steps. Furthermore, once the Gaussian widths have been fixed (which may be an approximate process), PES information is needed only at the Gaussian centers $\mathbf{Q}_{i}$.

The BEL MCG method will be available in the development version of the Heidelberg MCTDH package.

The authors would like to thank Graham Worth for fruitful discussions and valuable insights into the intricacies of vMCG.

*wkoch@rsc.anu.edu.au †jf@

[1] R. Kosloff, Annu. Rev. Phys. Chem. 45, 145 (1994).

[2] N. Makri, Annu. Rev. Phys. Chem. 50, 167 (1999).

[3] M. Yang, J. Chem. Phys. 129, 064315 (2008).

[4] J. Sielk, H. F. von Horsten, F. Krüger, R. Schneider, and B. Hartke, Phys. Chem. Chem. Phys. 11, 463 (2009).

[5] A. Shimshovitz and D. J. Tannor, Phys. Rev. Lett. 109, 070402 (2012).

[6] H.-D. Meyer, U. Manthe, and L. Cederbaum, Chem. Phys. Lett. 165, 73 (1990).

[7] H.-D. Meyer, Wiley Interdiscip. Rev.: Comput. Mol. Sci. 2, 351 (2012).

[8] I. Burghardt, H.-D. Meyer, and L. S. Cederbaum, J. Chem. Phys. 111, 2927 (1999).
[9] G. A. Worth, M. A. Robb, and I. Burghardt, Faraday Discuss. 127, 307 (2004).

[10] B. Lasorne, M. J. Bearpark, M. A. Robb, and G. A. Worth, Chem. Phys. Lett. 432, 604 (2006).

[11] T. J. Frankcombe, M. A. Collins, and G. A. Worth, Chem. Phys. Lett. 489, 242 (2010).

[12] S.-I. Sawada, R. Heather, B. Jackson, and H. Metiu, J. Chem. Phys. 83, 3009 (1985).

[13] M. Ben-Nun and T. J. Martínez, J. Chem. Phys. 108, 7244 (1998).

[14] M. Ben-Nun, J. Quenneville, and T. J. Martínez, J. Phys. Chem. A 104, 5161 (2000).

[15] S. Mallat and Z. Zhang, IEEE Trans. Signal Process. 41, 3397 (1993).

[16] Y. Wu and V.S. Batista, J. Chem. Phys. 118, 6720 (2003).

[17] P. Dirac, Math. Proc. Cambridge Philos. Soc. 26, 376 (1930).

[18] J. Frenkel, Wave Mechanics: Advanced General Theory (Clarendon, Oxford, 1934), Vol. 2.

[19] T. J. Frankcombe and S.C. Smith, J. Theor. Comput. Chem. 02, 179 (2003).

[20] J. A. Nelder and R. Mead, Comput. J. 7, 308 (1965).

[21] C. Eckart, Phys. Rev. 35, 1303 (1930).

[22] U. Manthe, H.-D. Meyer, and L. S. Cederbaum, J. Chem. Phys. 97, 3199 (1992).

[23] B. Lasorne, M. A. Robb, and G. A. Worth, Phys. Chem. Chem. Phys. 9, 3210 (2007).

[24] B. T. Sutcliffe and R. G. Woolley, J. Chem. Phys. 137, 22A544 (2012). 